\title{
Evolución de los cuidados de las heridas quirúrgicas inguinales cerradas tras cirugía de endoprótesis o bypass arterial: una revisión narrativa
}

\author{
Patricia Carrasco Rodríguez ${ }^{1}$, Jaime Muñoz Castellanos ${ }^{1}$, Eugenia Barrio Martín ${ }^{2}$, Jesús De Cabo Calvo ${ }^{1}$, Francisco \\ José Granado Sánchez ${ }^{1}$, Araceli Jiménez Martínez ${ }^{1}$, Silvia Sanguino García ${ }^{1}$. \\ ${ }^{1}$ Enfermera/o Unidad de Cirugía Vascular. Hospital Universitario Ramón y Cajal. Madrid. España. \\ ${ }^{2}$ Supervisora Unidad de Cirugía Vascular. Hospital Universitario Ramón y Cajal. Madrid. España.
}

\author{
Correspondencia \\ Patricia Carrasco Rodríguez. \\ Correo electrónico: pcarrasco2005@gmail.com \\ (Recibido: 15/10/2018. Aceptado: 27/11/2018)
}

\begin{abstract}
RESUMEN
Las heridas quirúrgicas inguinales cerradas cobran especial importancia debido a su particular tendencia a la infección tras cirugías de bypass y endoprótesis arteriales. Las complicaciones postoperatorias se acentúan sobre todo en personas con dificultades para la eliminación y/o con diferentes comorbilidades. La terapia de presión negativa es una de las alternativas de reciente consideración para la prevención de la infección de las heridas quirúrgicas vasculares de origen inguinal. Nos propusimos realizar una revisión de la evidencia existente acerca del uso de la terapia de presión negativa en la prevención de las infecciones de las incisiones quirúrgicas inguinales durante el postoperatorio de cirugía vascular. Las bases bibliográficas utilizadas fueron Medline, Cinhal y Embase. Se obtuvieron ocho artículos, de los cuales tres se dedican exclusivamente al abordaje inguinal. Existen escasos estudios experimentales y de calidad que se dediquen específicamente a evaluar la eficacia de la terapia de presión negativa en la prevención de las infecciones en heridas quirúrgicas inguinales frente a otros tipos de curas.
\end{abstract}

\section{PALABRAS CLAVE}

Ingle; Terapia de heridas por presión negativa; Procedimientos quirúrgicos vasculares; Infecciones de heridas quirúrgicas

\section{SUMMARY}

Closed inguinal surgical wounds are especially important due to their particular tendency to infection after bypass and arterial stent surgeries. The postoperative complications are accentuated especially in people with difficulties with elimination and / or with different commodities. Negative pressure therapy is one of the alternatives recently considered for the prevention of infection of vascular surgical wounds of inguinal origin.

We proposed to review the existing evidence about the use of negative pressure therapy in the prevention of infections of the inguinal surgical incisions during the postoperative period of vascular surgery. The bibliographic bases used were Medline, Cinhal and Embase. Eight articles were obtained, three of which are dedicated exclusively to the inguinal approach. There are few experimental and quality studies specifically dedicated to evaluating the efficacy of negative pressure therapy in the prevention of infections in inguinal surgical wounds versus other types of cures.

\section{KEYWORDS}

Groin; Negative-pressure wound therapy; Vascular surgical procedures; Surgical wound infections

\section{Introducción}

Las infecciones de localización quirúrgica forman parte de las infecciones nosocomiales más frecuentes en la Comunidad de Madrid (1). La localización quirúrgica inguinal tiene unas peculiaridades determinadas: grosor disminuido de la epidermis, repliegues cutáneos que son mayores en el caso de personas obesas, proximidad con la zona genital, estructuras linfáticas. Estas particularidades contribuyen a incrementar su vulnerabilidad frente a otras localizaciones anatómicas. Las complicaciones postoperatorias se acentúan sobre todo en personas con dificultades para la eliminación y/o diferentes comorbilidades. Varios artículos publicados $(2,3,4)$ han destacado que la localización anatómica de estas heridas quirúrgicas, junto con la existencia de hematomas, seromas, linforrea, colonización por SARM, reintervención e implantación de prótesis constituyen importantes factores de riesgo que favorecen la infección postoperatoria. En el servicio de Cirugía Vascular del Hospital Universitario Ramón y Cajal los accesos quirúrgicos inguinales responden mayoritariamente a cirugías de bypass y endoprótesis arteriales. Según datos del servicio de Medicina Pre- 
ventiva del hospital, después de un repunte en el año 2015 de la tasa de incidencia acumulada de infección de herida quirúrgica en nuestro servicio, actualmente ésta se sitúa en torno a un $2,52 \%$, quedando por debajo de la media del hospital para ese tipo de infecciones.

La terapia de presión negativa (TPN) es una alternativa de reciente incorporación para el tratamiento de las heridas inguinales cerradas tras cirugía vascular con la finalidad de prevenir infecciones postquirúrgicas. Pocos organismos contemplan en sus guías esta nueva perspectiva preventiva, sin embargo, la OMS (Organización Mundial de la Salud) en sus indicaciones para la prevención de la infección del sitio quirúrgico (5), recomienda el uso de la TPN para la prevención de infecciones en las heridas quirúrgicas de alto riesgo en pacientes adultos a pesar de su baja evidencia.

Los profesionales de enfermería nos encontramos con el reto de prevenir las complicaciones postquirúrgicas de las incisiones inguinales, por lo que es importante planificar los cuidados en base a la mejor evidencia disponible. Además, el retraso en la cicatrización de los accesos quirúrgicos inguinales repercute negativamente en el aspecto laboral y psicosocial de las personas que lo sufren $y$, por añadidura, en el sistema sanitario público, encareciendo el gasto en recursos materiales y humanos (6).

En los últimos años ha habido un importante proceso evolutivo, no solo en cuanto al tratamiento, sino también en cuanto a la prevención de las complicaciones de las heridas quirúrgicas en que la zona de abordaje había sido una o ambas ingles. En este proceso la enfermería es una pieza clave en la prevención de las complicaciones quirúrgicas. El propósito de este estudio fue realizar una revisión de la literatura publicada acerca del uso de la TPN en la prevención de las infecciones de las incisiones quirúrgicas inguinales durante el postoperatorio de cirugía vascular. quirúrgico vascular (endoprótesis y bypass arterial) en los últimos 6 años (2012-2018), seleccionando únicamente estudios con metodología experimental, revisiones sistemáticas y metaanálisis. En el caso de las revisiones sistemáticas y los metaanálisis, se incluyeron estudios que, aunque no estuvieran exclusivamente centrados en las heridas inguinales, incluyesen en su universo muestral estudios sobre heridas inguinales.

\section{Desarrollo y discusión}

La mayor parte de los trabajos encontrados estaban centrados en la evaluación de la eficacia de la TPN en relación a otros tipos de curas convencionales realizadas con diferentes tipos de apósitos. Se encontraron escasos estudios centrados de forma exclusiva en heridas quirúrgicas inguinales cerradas de origen vascular. Finalmente se obtuvieron ocho artículos: cuatro eran ensayos clínicos aleatorizados, de los cuales tres se dedicaban exclusivamente al abordaje inguinal; uno era una revisión sistemática, y tres eran metaanálisis (Tabla 2).

Masden et al. (6) publican un ensayo clínico aleatorizado sobre el riesgo de infección en diversos tipos de incisiones quirúrgicas de riesgo (incluida la localización inguinal, entre otras), y concluyen que no hay diferencias significativas en cuanto al desarrollo de infecciones en personas con diferentes comorbilidades al comparar la TPN con apósitos de silicona y plata. Los autores atribuyen este resultado a la baja difusión de la TPN por aquellos años y a un tamaño muestral insuficiente.

Esto se ha realizado así, debido a la ausencia de revisiones sistemáticas y metaanálisis que contengan únicamente revisiones sobre la prevención de la infección de heridas inguinales cerradas y para comprender si realmente los autores que los realizan consideran la singularidad de la localización inguinal en relación a otras localizaciones quirúrgicas. Se excluyeron los estudios que no cumpliesen los requisitos previos de inclusión, que no estuviesen en

Tabla 1: Estrategias de búsqueda y artículos obtenidos.

\begin{tabular}{clc}
\hline $\begin{array}{c}\text { Base de } \\
\text { datos }\end{array}$ & \multicolumn{1}{c}{ Estrategia de Búsqueda } & Artículos obtenidos \\
\hline Cinahl & $\begin{array}{l}\text { (Vascular Surgery OR Embolectomy OR Endarterectomy OR } \\
\text { Thrombectomy OR Angioplasty OR Blood Vessel Prosthesis) AND } \\
\text { (Surgical Wound Care OR Negative Pressure Wound Therapy) AND } \\
\text { (Groin). }\end{array}$ & 5 \\
\hline Embase & $\begin{array}{l}\text { (Surgical Infection) AND (Vascular Surgery) AND } \\
\text { (Vacuum Assisted Closure) AND (Wound Dressing). }\end{array}$ & 24 \\
& $\begin{array}{l}\text { (Vascular Surgical Procedures) AND (Groin OR Inguinal Canal) AND } \\
\text { (Negative-Pressure Wound Therapy OR Wound Closure Tech- } \\
\text { niques). }\end{array}$ \\
\hline
\end{tabular}

\section{Material y métodos}

Se realizó una búsqueda bibliográfica y posterior revisión narrativa de estudios publicados sobre el uso de TPN en heridas inguinales cerradas tras procedimiento inglés o español, los realizados en animales, aquellos centrados en la población pediátrica y los que no tuviesen una metodología clara según las recomendaciones de la CASPe (Critical Appraisal Skills Programme en español). 
Las bases bibliográficas consultadas fueron Medline, Cinhal y Embase. Para formular las tres estrategias de búsqueda se utilizó la plataforma MeSH de Medline y los tesauros de Cinahl (Cinahl Headings) y Embase (Emtree) para convertir el lenguaje libre en lenguaje controlado (Tabla 1). Mediante este proceso se obtuvieron en total tres artículos. Posteriormente, debido a los escasos hallazgos, se amplió la búsqueda en Google Scholar utilizando las palabras clave para poder encontrar algún estudio más de calidad, y se obtuvieron cinco artículos más. se dedican exclusivamente a heridas cerradas inguinales. Los autores consideran que el efecto de la terapia de vacío en heridas inguinales es más importante que en heridas con otras localizaciones, y consideran que las incisiones localizadas en las ingles deben ser estudiadas por separado. También resaltan las diferentes comorbilidades de las personas que componen las muestras de los trabajos estudiados, lo cual es considerado como un tipo de sesgo por los autores.

Tabla 2: Descripción de los estudios resultantes del proceso de búsqueda.

\begin{tabular}{llc}
\hline Autores/Año publicación & Tipo de Estudio & $\begin{array}{c}\text { \% localizaciones } \\
\text { inguinales incluidas }\end{array}$ \\
\hline Masden et al. (2012) & Ensayo aleatorizado controlado & $4,97 \%$ \\
\hline Ingargiola et al. (2013) & Revisión sistemática & $10,38 \%$ \\
\hline Semsarzadeh et al. (2015) & Metaanálisis & $6,82 \%$ \\
\hline Strugala y Martin. (2017) & Metaanálisis & $0,55 \%$ \\
\hline Hyldig et al. (2016) & Revisión sistemática & $100 \%$ \\
\hline Pleger et al. (2018) & Estudio aleatorizado prospectivo & $100 \%$ \\
\hline Engelhardt et al. (2018) & Ensayo aleatorizado controlado & $100 \%$ \\
\hline Gombert et al. (2018) & Ensayo aleatorizado prospectivo & 105 \\
\hline
\end{tabular}

Ingargiola et al. (7) realizan una revisión sistemática en 2013 en la que no pueden concluir que el uso de la TPN en heridas cerradas de diferentes localizaciones anatómicas reduzca la tasa de infección y dehiscencia, debido a la variabilidad metodológica de los estudios incluidos y a las diferentes comorbilidades y riesgos de las poblaciones incluidas. Convienen que harían falta más estudios para dilucidar si la TPN ofrece una eficacia significativamente superior en la prevención de la infección postquirúrgica de la incisión inguinal frente a las otras terapias convencionales.

Hylding et al. (8) realizan un metaanálisis incluyendo únicamente ensayos clínicos controlados que evalúan la eficacia de la TPN frente a los apósitos habituales en diferentes localizaciones anatómicas, incluyendo la ingle. Llegan a la conclusión de que la TPN parece eficaz en la prevención de la infección y la formación de seroma, pero que sus resultado no son generalizables debido a la heterogeneidad de los estudios: hay gran variabilidad en las intervenciones que se comparan (aparatos de vacío utilizados con distintos tipos de materiales y presiones aplicadas), en los tipos de incisiones y en las comorbilidades de las poblaciones que componen el universo de los estudios.

Semsarzadeh et al. (9) llevan a cabo un metaanálisis en el que llegan a la conclusión de que la TPN reduce la probabilidad de infección, dehiscencia y linforrea en heridas quirúrgicas cerradas en comparación con otros tipos de curas. Entre los estudios incluidos, tres de ellos
En 2017, Strugala y Martin (10) realizan un nuevo metaanálisis con la intención de que los estudios incluidos en la revisión comparasen exclusivamente la TPN PICO ${ }^{\circledR}$ (Smith \& Nephew) con la utilización de apósitos en varios tipos de localizaciones anatómicas, entre las que se incluye la ingle. Los autores concluyen que esta terapia aporta muchas ventajas relativas a la disminución de la infección, la dehiscencia y los días de permanencia en el hospital, pero ellos mismos reconocen que, a pesar de haber querido reducir la heterogeneidad de las intervenciones seleccionando estudios que evalúan exclusivamente la TPN de esa casa comercial, existe variabilidad en las localizaciones anatómicas de las incisiones, en las comorbilidades de la población seleccionada y en la orientación metodológica de los estudios comprendidos.

Engelhardt et al. (11) publican en 2018 un ensayo aleatorizado en el que se compara la eficacia de la TPN frente a un apósito absorbente en la prevención de la infección en heridas quirúrgicas inguinales. Según los autores, se trata del primer estudio que estudia exclusivamente una muestra de personas sometidas a cirugía de reconstrucción arterial. Además, los grupos intervención y control son más o menos homogéneos, pero los autores no pueden confirmar estadísticamente si la TPN previene la infección de la incisión quirúrgica inguinal cerrada, aunque aprecian una ligera tendencia a favor de la misma. Esto lo atribuyen a una muestra 
insuficiente y consideran que hacen falta más estudios exclusivos.

En el mismo año, Gombert et al. (12) realizan un estudio similar y de las mismas características metodológicas. En este caso, la diferencia con el estudio previo estriba en que la muestra es mayor, aunque los grupos intervención y control se caracterizan por carecer de homogeneidad en un aspecto determinado: el análisis desvela que en el grupo de intervención (el de la TPN) las tasas de infección de la herida quirúrgica se ven disminuidas, y los autores lo relacionan con la baja predisposición a la infección de las personas de este grupo, según la clasificación de Szilagyi, con respecto al grupo control en el que el riesgo de infección era inicialmente más elevado. Otro aspecto destacado en cuanto a limitaciones del ensayo se centra en la falta de cegamiento que, aunque difícil, según estos autores sería necesaria para reducir sesgos.

Pleger et al. (13) concluyen en otro ensayo aleatorizado y controlado que la TPN es más eficaz que un apósito convencional en la reducción de las complicaciones en las heridas quirúrgicas inguinales vasculares, reduciéndose su efectividad a partir del séptimo día de su uso. Se trata de un ensayo en el que los grupos intervención y control son homogéneos y muy similares en número. También destacan al final del estudio que el tipo de terapia utilizada (Prevena Therapy ${ }^{\circledR}$ ) debería ser comparada con PICO ${ }^{\circledR}$ por ser diferente el mecanismo de gestión del exudado y la presión negativa que ejerce cada una.

Se ha podido apreciar que una de las tendencias actuales en el uso de TPN se encuentra en el campo de la prevención de infecciones de heridas quirúrgicas inguinales en el postoperatorio inmediato de intervenciones vasculares.

Como resultado de la búsqueda bibliográfica realizada se hallaron estudios longitudinales (14) y observacionales retrospectivos $(15,16)$ y prospectivos $(17,18)$, pero escasos estudios experimentales y de calidad. Esto podría deberse al hecho de que esta indicación preventiva de la TPN sea una perspectiva relativamente reciente.

En cuanto a la baja evidencia sobre la eficacia de la TPN en heridas quirúrgicas inguinales cerradas, debemos decir que no ha variado mucho en el tiempo. Una revisión Cochrane de 2008 (19) sugería ya los beneficios de la misma en heridas crónicas, pero sin recomendar su uso por la necesidad de más estudios. Ocurrió lo mismo en el caso de las curas en heridas por primera intención (20) y segunda intención (21). Sin embargo, otra revisión Cochrane de 2013 (22), reconocía cierta evidencia en la eficacia del uso de la TPN en heridas de pie diabético. Siguiendo la estela de este último estudio, una tendencia homogeneizadora en cuanto a comorbilidades y otros aspectos en la elección de la muestra participante sería beneficiosa para la producción de estudios de mayor calidad que aporten evidencia al respecto. Esto coincide con la perspectiva de varios de los autores aquí analizados, que apuntan a los distintos tipos de comorbilidades de los sujetos que componen las muestras seleccionadas como responsables de los sesgos de los estudios.

También hay que tener en cuenta que, a menudo, las marcas comerciales empleadas, los procedimientos de cura y/o los componentes utilizados en los grupos control son diferentes en cada estudio. Por otra parte, en la práctica diaria a veces es preciso conciliar el uso de TPN con otros tipos de terapias, debido, por ejemplo, a la existencia de colonias de microorganismos: existen antisépticos como la polihexanida que pueden ser utilizados en la prevención de la infección de localización quirúrgica solos o al mismo tiempo que la TPN en pacientes con SARM en cultivos previos (23). Todos los factores mencionados podrían dificultar o impedir que se obtengan conclusiones firmes en los estudios existentes.

La limitación principal de este estudio radica en el bajo nivel de evidencia inherente a toda revisión narrativa. Además, los resultados obtenidos han sido muy escasos y poco determinantes.

Sería conveniente que la comunidad científica se hiciese eco de las demandas de la OMS, que pide que se lleven a cabo más estudios sobre la eficacia de la TPN en la prevención de la infección del sitio quirúrgico y que ayuden a determinar sobre qué grupos de pacientes esta intervención sería más coste-efectiva. Desde otra perspectiva, se necesitan estudios que determinen si la disminución del tiempo de ingreso podría ser atribuible al uso de la TPN, que aclaren qué otros recursos concretos se verían optimizados y que valoren la satisfacción de los pacientes así como otros aspectos psicoemocionales.

El análisis de todos estos factores podría ser de gran ayuda a la hora de elegir el tratamiento preventivo de las infecciones de las heridas quirúrgicas de localización inguinal de la manera más coste-efectiva posible.

\section{Conclusiones}

Una de las tendencias actuales en la prevención de infecciones de las incisiones quirúrgicas inguinales cerradas es el uso de TPN, pero hay escasos estudios experimentales de calidad que se dediquen específicamente a evaluar su eficacia frente a otros tipos de curas.

Por lo tanto, para poder hacer recomendaciones sobre su uso sería necesario realizar más estudios de calidad. Sería necesario que esos estudios mantuviesen la máxima homogeneidad posible tanto en los criterios de selección de las muestras como en las intervenciones a comparar, y que analizasen tanto la costoeficiencia de los materiales empleados como la satisfacción de los pacientes o cualquier otro factor que pueda ser de ayuda en la toma de decisiones.

Conflicto de intereses: Ninguno. 


\section{Bibliografía}

1. Díaz-Agero-Pérez C, Pita-López MJ, RobustilloRodela A, Figuerola-Tejerina A, Monge-Jodrá V, Grupo de Trabajo INCLIMECC de la Comunidad de Madrid. Evaluación de la infección de herida quirúrgica en 14 hospitales de la Comunidad de Madrid: estudio de incidencia. Enferm Infecc Microbiol Clin. 2011; 29(4):257-262.

https://doi.org/10.1016/ j.eimc.2010.09.009.

2. Slappy AL, Hakaim AG, Oldenburg WA, PazFumagalli R, McKinney JM. Femoral incision morbidity following endovascular aortic aneurysm repair. Vasc Endovasc Surg. 2003; 37:105-9. https://doi.org/10.1177/153857440303700204.

3. Bandyk DF. Vascular surgical site infection: risk factors and preventive measures. Seminars in Vascular Surgery. 2008; 21(3):119-123.https://doi.org/ 10.1053/j.semvascsurg.2008.05.008.

4. Ploeg AJ, Lardenoye J-WP, Vrancken Peeters MPFM, Hamming JF, Breslau PJ. Wound complications at the groin after peripheral arterial surgery sparing the lymphatic tissue: a double-blind randomized clinical trial. American Journal of Surgery. 2009; 197(6):747-751. https://doi.org/10.1016/j.amjsurg. 2008.04.014.

5. WHO Guidelines Approved by the Guidelines Review Committee. Global guidelines for the prevention of surgical site infection. Geneva: World Health Organization. 2016.

http://www.who.int/gpsc/global-guidelinesweb.pdf?ua=1.

6. De Lissovoy G, Fraeman K, Hutchins V, Murphy D, Song D, Vaughn BB. Surgical site infection: incidence and impact on hospital utilization and treatment costs. Am J Infect Control. 2009; 37(5):387-397. https://doi.org/10.1016/j.ajic.2008.12.010.

7. Masden D, Goldstein J, Endara M, Xu K, Steinberg J, Attinger $C$. Negative pressure wound therapy for atrisk surgical closures in patients with multiple comorbidities: a prospective randomized controlled study. Ann Surg. 2012; 255(6):1043-7. doi: 10.1097/ SLA.0b013e3182501bae.

8. Ingargiola MJ, Daniali LN, Lee ES. Does the Application of Incisional Negative Pressure Therapy to High-Risk Wounds Prevent Surgical Site Complications? A Systematic Review. Eplasty. 2013; 13:e49.

9. Hyldig N, Birke-Sorensen $H$, Kruse $M$, Vinter $C$, Joergensen JS, Sorensen JA et al. Meta-analysis of negative-pressure wound therapy for closed surgical incisions. BrJ Surg. 2016; 103: 477-486. https://doi.org/10.1002/bjs.10084.

10. Semsarzadeh N, Tadisina K, Maddox J, Chopra K, Singh $\mathrm{D}$. Closed incision negative-pressure therapy is associated with decreased surgical-site infections: a meta-analysis. Plast Reconstr Surg. 2015; 136: 592-602.

11. Strugala V, Martin R. Meta-analysis of comparative trials evaluating a prophylactic single-use negative pressure wound therapy system for the prevention of surgical site complications. Surg Infect. 2017; 18: 810-9. https://doi.org/10.1089/sur.2017.156.

12. Engelhardt M, Rashad NA, Willy $C$, Müller $C$, Bauer $C$, Debus $\mathrm{S}$, et al. Closed-incision negative pressure therapy to reduce groin wound infections in vascular surgery: a randomised controlled trial. Int Wound J. 2018; 15:327-332. doi: 10.1111/ iwj.1284.

13. Gombert A, Babilon M, Barbati M E, Keszei von Trotha K T, Jalaie $H$ et al. Closed Incision Negative Pressure Therapy Reduces Surgical Site Infections in Vascular Surgery: A Prospective Randomised Trial (AIMS Trial). European Journal of Vascular and Endovascular Surgery. 2018, 56(3): 442-448. DOI: 10.1016/J.EJVS.2018.05.018.

14. Pleger SP, Nink N, Elzien M, Kunold A, Koshty A, Böning $A$. Reduction of groin wound complications in vascular surgery patients using closed incision negative pressure therapy (ciNPT): a prospective, randomised, single-institution study. Int Wound J. 2018; 15:75-83. doi: 10.1111/iwj.1283.

15. Matatov T, Reddy KN, Doucet LD, Zhao CX, Zhang WW. Experience with a new negative pressure incision management system in prevention of groin wound infection in vascular surgery patients. 2013; J Vasc Surg 57:791-795. https://doi.org/10.1016/ j.jvs.2012.09.037.

16. Andersson S, Monsen C, Acosta S. Outcome and complications using negative pressure wound therapy in the groin for perivascular surgical site infections after vascular surgery. Annals of Vascular Surgery .2018; 48: 104-110. doi:10.1016/j.avsg.2017. 10.018 .

17. Zamani N, Sharath SE, Vo E, Awad S, Kougias $P$, Barshes NR. A Multi-Component Strategy to Decrease Wound Complications after Open InfraInguinal Re-Vascularization. Surgical Infections. 2018; 19 (1). http://doi.org/10.1089/sur.2017.193.

18. Fleming CA, Kuteva $M, O^{\prime}$ Hanlon $K, O^{\prime} B$ rien $G$, McGreal G. Routine use of PICO dressings may reduce overall groin wound complication rates following peripheral vascular surgery. Journal of Hospital Infection. 2018; 99:75e80. https://doi.org/10.1016/ j.jhin.2017.10.022.

19. Pesonen LO, Halloran BG. Aziz A. Prophylactic Groin Wound Vacuum-assisted Therapy in Vascular Surgery Patients at Enhanced Risk for Postoperative Wound Infection. Annals of Vascular Surgery. 2018; 46:127-133. DOI: 10.1016/J.AVSG.2017.07.009.

20. Ubbink DT, Westerbos SJ, Evans D, Land L, Vermeulen $\mathrm{H}$. Topical negative pressure for treating chronic wounds. Cochrane Database of Systematic Reviews. 2008; Issue 3. Art. No.: CD001898. DOI: 10.1002/ 14651858.CD001898.

21. Webster J, Scuffham P, Stankiewicz M, Chaboyer WP. Negative pressure wound therapy for skin grafts and surgical wounds healing by primary in- 
tention. Cochrane Database of Systematic Reviews 2014, Issue 10. Art. No.: CD009261. DOI: 10.1002/ 14651858.CD009261.pub3.

22. Dumville JC, Owens GL, Crosbie EJ, Peinemann F, Liu Z. Negative pressure wound therapy for treating surgical wounds healing by secondary intention. Cochrane Database of Systematic Reviews. 2015; Issue 6. Art. No.: CD011278. DOI: 10.1002/ 14651858.CD011278.pub2.
23. Dumville JC, Hinchliffe RJ, Cullum N, Game F, Stubbs $\mathrm{N}$, Sweeting M. Negative pressure wound therapy for treating foot wounds in people with diabetes mellitus. Cochrane Database of Systematic Reviews. 2013; Issue 1. Art. No.: CD010318. DOI: 10.1002/ 14651858.CD010318

24. Kramer A, Dissemond J, Kim S, Willy C, Mayer D, Papke $R$, et al. Consensus on wound antisepsis: update 2018. Skin Pharmacol Physiol. 2017; 31: 28-58. https://doi.org/10.1159/000481545. 